\title{
Funcionalidad Familiar e Indicadores Emocionales en Escolares, Institución Educativa Integral "Blas Valera Pérez" Chachapoyas- 2017
}

\section{Family Functionality and Emotional Indicators in Schoolchildren, Integral Educational Institution "Blas Valera Pérez" Chachapoyas- 2017}

\author{
Gladiceidy Alva Salazar ${ }^{1}$ - Sonia Tejada Muñoz
}

\begin{abstract}
RESUMEN
La presente investigación se realizó con el objetivo de determinar la relación que existe entre la funcionalidad familiar e indicadores emocionales en escolares, Institución Educativa Integral "Blas Valera Pérez" Chachapoyas, 2017; fue de enfoque cuantitativo; de nivel relacional, observacional, prospectivo, transversal, analítico. El universo muestral estuvo conformado por 33 niños escolares que fluctuan entre 6 y 12 años y sus padres. Para la recolección de datos se utilizó el "Test de Funcionamiento Familiar FF - SIL" López Nicolás (2003), con una confiabilidad de 0.93 y validez de 3.82, y el test del dibujo de la Figura Humana (DFH) de Koppitz con una confiablidad de 0,77 y validez de 0,51. Del 100 \% (33) de los escolares, Institución Educativa Blas Valera Pérez, Chachapoyas, el 39.4\% (13) presentan indicadores emocionales de nivel bajo y viven en familias moderadamente funcionales, el $33.3 \%$ (11) presenta indicadores emocionales de nivel bajo y viven en familias disfuncionales. El $24.2 \%$ (8) presentan indicadores emocionales de nivel bajo y viven en familias funcionales. Solo el $3 \%$ (1) no presento ningún indicador emocional y viven en familias moderadamente funcionales. Conclusión: No existe relación estadistica entre funcionalidad familiar e indicadores emocionales en los escolares en la institución educativa integral "Blas Valera Perez" Chachapoyas. $\left(X^{2}=5.135 ; \mathrm{gl}=10 ; \mathrm{p}=0.882>0.05\right)$
\end{abstract}

Palabras clave: Funcionalidad familiar, indicadores emocionales, escolares.

\begin{abstract}
The present research was carried out with the objective of determining the relationship that exists between family functionality and emotional indicators in schoolchildren in the Integral Educational Institution "Blas Valera Pérez" Chachapoyas, 2017; it was a quantitative approach; of relational, observational, prospective, transversal, analytic level. The sample universe consisted of 33 school children that fluctuated between 6 and 12 years old and their parents. For the data collection, the "Family Function Test FF - SIL" López Nicolás (2003) was used, with a reliability of 0.93 and validity of 3.82, and the Human Figure drawing (DFH) test of Koppitz with a reliability of 0.77 and validity of 0.51 . The results $39.4 \%$ (13) present low-level emotional indicators and live in moderately functional families, 33.3\% (11) present low-level emotional indicators and live in dysfunctional families. 24.2\% (8) present emotional indicators of low level and live in functional families. Only 3\% (1) did not present any emotional indicators and live in moderately functional families. Conclusion: There is no statistical relationship between family functionality and emotional indicators in school children in the comprehensive educational institution "Blas Valera Perez" Chachapoyas. $\left(\mathrm{X}^{2}=5.135, \mathrm{gl}=2, \mathrm{p}=0.882>0.05\right)$
\end{abstract}

Keywords: Family functionality, emotional indicators, school.

\footnotetext{
${ }^{1}$ Licenciada en Enfermería. Egresada de la Escuela Profesional de Enfermería de la Universidad Nacional Toribio Rodríguez de Mendoza de Amazonas.Email: Ceidy.almh@gmail.com

${ }^{2}$ Dra en Ciencias de Enfermería, asociada al departamento de Salud Pública a TC- UNTRM-Amazonas. Email: cieloceleste120@hotmail.com
} 


\section{INTRODUCCIÓN}

La funcionalidad familiar es la capacidad del sistema para enfrentar y superar cada una de las etapas del ciclo vital y las crisis por las que atraviesa. Esto significa que debe cumplir con las tareas encomendadas, que los hijos no presenten trastornos graves de conducta y que la pareja no esté en lucha constante (Valle, 2014, p.1).

El funcionamiento en la familia es un factor determinante en la conservación de la salud o en la aparición de la enfermedad entre sus miembros. Con base en que la familia cumpla o deje de cumplir eficazmente sus funciones, se habla de familia funcional o disfuncional, ya que la funcionalidad familiar es la capacidad del sistema para enfrentar y superar cada una de las etapas del ciclo vital y las crisis por las que atraviesa (Rangel, 2014, p. 24).

Para Koppitz (1995) los Indicadores emocionales (IE) son signos clínicos que reflejan actitudes y características subyacentes de los niños en el momento de realizar sus DFH. Los IE revelan sentimientos y preocupaciones iguales o similares, y una misma actitud puede ser expresada por diversos IE. Señala que la presencia de dos o más indicadores emocionales es altamente sugestiva de problemas emocionales y relaciones personales insatisfactorias. (Henao, 2006, p. 10)

Se calcula que aproximadamente el $20 \%$ de los niños y adolescentes del mundo tienen trastornos o problemas mentales. Cerca de la mitad de los trastornos mentales se manifiestan antes de los 14 años. En todas las culturas se observan tipos de trastornos similares. Los trastornos neuropsiquiátricos figuran entre las principales causas de discapacidad entre los jóvenes. Sin embargo, las regiones del mundo con los porcentajes más altos de población menor de 19 años son las que disponen de menos recursos de salud mental. La mayoría de los países de ingresos bajos y medios cuenta con un solo psiquiatra infantil por cada millón a cuatro millones de personas. (Organización Mundial de la Salud, 2017,p.1)

El dibujo ha sido considerado un sistema de comunicación natural del niño, por lo que los test derivados de esta actividad han tenido rápida $\mathrm{y}$ extensa utilización. La capacidad del dibujo para expresar las emociones infantiles tiene una larga historia de investigación. (Koppitz, 1974) comenta que los sentimientos que un niño no desea o es incapaz de traducir en palabras o los sentimientos demasiado fuertes o confusos para ser pensados con claridad pueden expresarlos con mayor facilidad a través de sus dibujos o pinturas. (Garaigordobil y
Maganto, 2012, p.76)

En nuestro medio en los últimos años, diversas instituciones educativas y de salud han dado conocer cifras estadísticas que demuestran una alta incidencia de indicadores emocionales alterados en la etapa escolar; situaciones poco reconocidos como factores determinantes o prevenibles en los diferentes ámbitos: familia, comunidad y escuela; por lo que se considera pertinente identificar la relación de dichos indicadores con la funcionalidad familiar en la Institución Educativa Blas Valera Pérez de Chachapoyas, Amazonas; a través de mecanismos objetivos de identificación y que permiten ser atendidos de manera oportuna y pertinente, fortaleciendo las capacidades de la familia y de los y las educadores/as, potenciando las capacidades integrales de los niños, niñas y actuando para que los mismos no sea afectados en mayor magnitud o se coloque en una situación de vulnerabilidad que haga más complejo o difícil recuperar su situación.

Se considera un aporte valioso para la enfermería escolar que permita ampliar su rol laboral poco explorado en esta área y también sensibilice a autoridades para proponer políticas educativas públicas nacionales y regionales en el marco de una formación y educación integral.

En este contexto se formuló la siguiente interrogante: ¿Existe relación entre la funcionalidad familiar e indicadores emocionales en escolares, Institución Educativa Integral "Blas Valera Pérez" Chachapoyas, 2017.?

\section{MATERIAL Y MÉTODOS}

Fue de enfoque cuantitativo; de nivel relacional; de tipo de investigación: tipo observacional; según la planificación de la toma de datos es prospectivo, según el nivel de medición de las variables es de tipo transversal y según el nivel de interés de las variables estadísticas es analíticas.

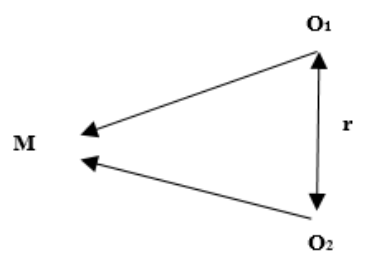

Dónde:

$\mathrm{M}=$ niños escolares y su respectivo padres

$\mathrm{r}=$ relación de las variables

$\mathrm{O}_{1}=$ funcionalidad familiar

$\mathrm{O}_{2}=$ indicadores emocionales

Para la población muestral estuvo constituido por 33 
niños escolares que fluctuan entre 6 y 12 años y sus padres: matriculados en el año 2017, en la Institución Educativa Integral Blas Valera Pérez; Para medir la variables funcionalidad familiar se usó; el método de la encuesta y la técnica el cuestionario "Test de Funcionamiento Familiar FF - SIL"; Para la variable indicadores emocionales se usó el método de la encuesta y la tecnica de la psicometría, el test del dibujo de la Figura Humana (DFH). Se utilizó la estadística descriptiva e inferencial Ji-cuadrado y se procesaron los datos en el SPSS V-21.

\section{RESULTADOS}

Tabla 01: Funcionalidad familiar de los escolares, Institución Educativa Blas Valera Pérez, Chachapoyas, 2017

\begin{tabular}{lcc}
\hline Funcionalidad familiar & fi & $\%$ \\
\hline Severamente Disfuncional & 0 & 0 \\
Disfuncional & 11 & 33.3 \\
Moderadamente Funcional & 14 & 42.4 \\
Funcional & 8 & 24.2 \\
\hline Total & $\mathbf{3 3}$ & 100.00 \\
\hline
\end{tabular}

Del $100 \%$ (33) de los escolares, Institución Educativa Blas Valera Pérez, se evidencia que el $42.4 \%$ (14) viven dentro de las familias moderadamente funcionales, el $33.3 \%$ (11) en familias disfuncionales y $24.2 \%$ (8) en familias funcionales.

Tabla 02: Indicadores emocionales de los escolares, Institución Educativa Blas Valera Pérez, Chachapoyas, 2017

\begin{tabular}{lcc}
\hline Indicador emocional & fi & $\%$ \\
\hline Alto & 0 & 0 \\
Medio & 0 & 0 \\
Bajo & 32 & 97 \\
Ninguno & 1 & 3 \\
\hline Total & $\mathbf{3 3}$ & 100 \\
\hline Fuente: Encuesta de test de la figura humana, aplicado. & &
\end{tabular}

Del $100 \%$ (33) de los escolares, Institución Educativa Blas Valera Pérez, Chachapoyas, tienen indicadores emocionales con un $97 \%$ (32) nivel bajo, y el $3 \%$ (1) no tiene ningún indicador. No existiendo estudiantes con indicadores emocionales negativos de nivel medio y alto respectivamente.
Tabla 03: Funcionalidad familiar e indicadores emocionales de los escolares, Institución Educativa Blas Valera Pérez, Chachapoyas, 2017

\begin{tabular}{|c|c|c|c|c|c|c|c|c|c|c|}
\hline \multirow{3}{*}{$\begin{array}{l}\text { Funcionalidad } \\
\text { familiar }\end{array}$} & \multicolumn{8}{|c|}{ Indicadores emocionales } & \multirow{2}{*}{\multicolumn{2}{|c|}{ Total }} \\
\hline & \multicolumn{2}{|c|}{ Alto } & \multicolumn{2}{|c|}{ Medio } & \multicolumn{2}{|c|}{ Bajo } & \multicolumn{2}{|c|}{ Ninguno } & & \\
\hline & fi & $\%$ & fi & $\%$ & fi & $\%$ & fi & $\%$ & fi & $\%$ \\
\hline $\begin{array}{l}\text { Severamente } \\
\text { disfuncional }\end{array}$ & 0 & 0 & 0 & 0 & 0 & 0 & 0 & 0 & 0 & 0 \\
\hline Disfuncional & 0 & 0 & 0 & 0 & 11 & 33.3 & 0 & 0 & 11 & 33.3 \\
\hline $\begin{array}{l}\text { Moderadamente } \\
\text { funcional }\end{array}$ & 0 & 0 & 0 & 0 & 13 & 39.4 & 1 & 3 & 14 & 42.4 \\
\hline Funcional & 0 & 0 & 0 & 0 & 8 & 24.2 & 0 & 0 & 8 & 24.2 \\
\hline Total & 0 & 0 & 0 & 0 & 32 & 97.0 & 1 & 3 & 33 & 100.0 \\
\hline
\end{tabular}

Del $100 \%$ (33) de los escolares, Institución Educativa Blas Valera Pérez, Chachapoyas, el 39.4 $\%$ (13) presentan indicadores emocionales de nivel bajo y viven en familias moderadamente funcionales, el $33.3 \%$ (11) presenta indicadores emocionales de nivel bajo y viven en familias disfuncionales. El 24.2 $\%$ (8) presentan indicadores emocionales de nivel bajo y viven en familias funcionales.

Solo el $3 \%$ (1) no presento ningún indicador emocional y viven en familias moderadamente funcionales.

\section{DISCUSIÓN}

En la tabla 01, se observa que del $100 \%$ (33) de los escolares, Institución Educativa Blas Valera Pérez, se evidencia que el $42.4 \%$ (14) viven dentro de las familias moderadamente funcionales, el $33.3 \%$ (11) en familias disfuncionales y $24.2 \%$ (8) en familias funcionales. De estos resultados se puede indicar que las decisiones, el cumplimiento de responsabilidad, muestras de cariño, el poder hablar de diversos temas sin temor y resolver problemas conjuntamente se realizan relativamente entre todos los familiares para cosas importantes que atañen a la familia.

Al respecto Clavijo et al (2016) en su estudio realizado en Colombia encontró que el $50.5 \%$ (192) de los escolares en estudio tuvieron una buena funcionalidad familiar, el $44.7 \%$ (170) tiene disfuncionalidad familiar, y el 4,7\% (18) tiene una disfuncionalidad familiar severa. Asimismo Bolaño, Cortina y Vargas (2013) indican que los resultados de la escala de funcionalidad familiar aplicado a escolares evidencian que el $81,9 \%$ de las familias presentan bajo nivel de funcionalidad familiar e intermedio un $18,1 \%$.

Según Graza (2013) en una investigación 
desarrollada en una institución educativa de Lima, encontró que un $39 \%$ de adolescentes provienen de familias disfuncionales, infiriéndose que una negativa funcionalidad familiar es una de las causas para la existencia de violencia escolar en los adolescentes. De igual manera Villalobos (2009) reveló funcionalidad familiar predominante de nivel bajo (52\%) seguido del nivel intermedio (47\%) y con un escaso nivel alto de funcionalidad $(2 \%)$.

Los resultados encontrados en la presente investigación son diferentes a los estudios de Clavijo et al (2016), Bolaño, Cortina y Vargas (2013), y Villalobos (2009) realizados en diferentes contextos, en donde se pone de evidencia que en los resultados predomina la funcionalidad familiar, seguidamente de una funcionalidad moderada, y con un menor porcentaje una disfunción. Mientras que con el estudio de Graza (2013) encontramos una similitud en los resultados.

Considerando lo mencionado anteriormente se debe resaltar según Castellón y Ledesma (2012) que el funcionamiento familiar es, por concepto, el conjunto de relaciones interpersonales que se generan en el interior de cada familia y que le confieren identidad propia. Asimismo Zaldívar (s.f) un funcionamiento familiar saludable es aquel que le posibilita a la familia cumplir exitosamente con los objetivos y funciones que le están histórica y socialmente asignados.

Las familias juegan un importante papel en el desarrollo de las habilidades sociales de sus hijas e hijos a través de su interactuar diario, y el ambiente donde sus hijos se van desarrollando va influir en su crecimiento como personas.

Cuando las relaciones familiares se caracterizan por una adecuada cohesión, adaptabilidad y comunicación, se tendrá un buen funcionamiento familiar ya que la socialización familiar fija una serie de conductas en los niños, al cual es probable que los que estan dentro de ello sean personas con una buena estabilidad emocional en el futuro. Por el contrario, cuando las familias son moderadamente funcionales y/o disfuncionales y los niños cerecen en un ambiente inadecuado por la presencia de conflicto, carencia de diálogo y deficiente apoyo a los miembros de la familia, pueden surgir problemas en los niños como, por ejemplo, problemas emocionales llevandoles a conductas inadaptables o incorrectas y dificultad en su aprendizaje, por lo tanto los padres de familia deben estar atentos para prevenir estos posibles riesgos de comportamniento en sus niños.

En la tabla 02, se observa que del $100 \%$ (33) de los escolares, Institución Educativa Blas Valera Pérez,
Chachapoyas, tienen indicadores emocionales con un $97 \%$ (32) nivel bajo, y el $3 \%$ (1) no tiene ningún indicador. No existiendo estudiantes con indicadores emocionales negativos de nivel medio y alto respectivamente. No existiendo estudiantes con indicadores emocionales negativos de nivel medio y alto. De estos resultados se puede evidenciar que se encontró dibujos de la figura humana pequeñas, interpretación pobre de las partes de la figura, con omisión de la nariz, brazos pegados al cuerpo y/o sombreados.

Existe similitudes con el estudio realizado por Mio (2016) en instituciones educativas de nivel inicial de Chachapoyas, ya que los niños en su mayoría presentaron indicadores emocionales negativos de medio a bajo respectivamente (I.E. Yance: $52.2 \%$ y 43,5\%; I.E. Divino Niño Jesús: $0 \%$ y 90.5\%; I.E. Santo Toribio de Mogrovejo: 41,7\% y 33,3\%).

De otro modo Castillo (2012) en una investigación desarrollada en una institución educativa de Chachapoyas, encontró indicadores emocionales negativos en niños, entre los cuales sobresalen los niveles bajo y medio. Según la dimensión impulsividad en su mayoría presentó un nivel medio. Asimismo en las dimensiones: timidez, ansiedad y agresividad se encuentran en un nivel medio, mientras que la inseguridad fluctúa en el nivel bajo.

Al respecto Ramírez, (2012) en su estudio realizado en Colombia encontró, que el $89 \%$ de escolares presenta indicadores de malestar emocional; en el trastorno con déficit de atención/hiperactividad (TDAH) tipo combinado la categoría más predominante frente a los indicadores emocionales, corresponde a la timidez, igual que para el TDAH hiperactivo impulsivo; finalmente para el TDAH Tipo inatento la categoría destacada fue la de pobre autoconcepto. Este porcentaje significativo de presencia de indicadores de malestar emocional en niños y niñas con diagnóstico de TDAH, pueden considerase de vital importancia, a la hora de reorientar las estrategias de intervención y tratamiento.

Los resultados encontrados en la presente investigación son similares a los estudios de Mío (2016), Castillo (2012), realizados en diferentes contextos, en donde se pone de evidencia que los escolares tienen problemas emocionales que están sugestivos a conductas perturbadoras. Mientras que Ramírez, (2012) nos presenta que la mayor población evaluada presenta indicadores de malestar emocional; en el trastorno con déficit de atención/hiperactividad (TDAH) tipo combinado la categoría más predominante frente a los indicadores emocionales. 
Los indicadores emocionales nos permiten analizar los sentimientos y emociones de los niños a través del dibujo de la figura humana se ha convertido en una técnica más usada por los profesionales para trabajar con los niños Koppitz, (1995) señala que la presencia de un solo indicador emocional no es concluyente y no es necesariamente un signo de perturbación emocional, sin embargo, dos o más indicadores emocionales son altamente sugestivos de problemas emocionales y relaciones personales insatisfactorias.

Es preocupante apreciar los resultados de los indicadores emocionales y se encuentra que el mayor porcentaje de escolares se encuentran en un nivel bajo, ya que estos indicadores emocionales nos podrían referir algunos signos clínicos que pueden reflejar actitudes y características inadecuadas que los niños lo pueden reflejar al momento de ejecutar sus dibujos de la figura humana. Por lo tanto es recomendable trabajar más con esta población para captar a tiempo si existiese un indicador emocional que estaría afectando en su desarrollo y consecuentemente en sus relaciones personales en un futuro.

Según resultados de la tabla 03 , del 100 \% (33) de los escolares, Institución Educativa Blas Valera Pérez, Chachapoyas, el 39.4 \% (13) presentan indicadores emocionales de nivel bajo y viven en familias moderadamente funcionales, el $33.3 \%$ (11) presenta indicadores emocionales de nivel bajo y viven en familias disfuncionales. El $24.2 \%$ (8) presentan indicadores emocionales de nivel bajo y viven en familias funcionales.

Solo el $3 \%$ (1) no presentó ningún indicador emocional y viven en familias moderadamente funcionales. La prueba estadística no paramétrica ji ${ }^{2}$ - cuadrado aplicado al estudio $\mathrm{X}^{2}=5.135 ; \mathrm{gl}=2 ; \mathrm{p}$ $=0.882>0.05$ no existe relación estadistica entre las variables.

No se han encontrado estudios sobre la relación entre funcionalidad familiar e indicadores emocionales, sin embargo algunas investigaciones relacionadas aportan algunos elementos para la discusión, tal es el caso del estudio de Lescano y Delgado (2012) quien identificó que no existe relación entre el funcionamiento familiar y el nivel de rendimiento académico de los estudiantes de 4to y 5 to grados del nivel secundario de la Institución Educativa N ${ }^{\circ} 0292$ del Distrito de Tabalosos, Tarapoto).

Al respecto, Moreno y Chauta (2011), en su investigación afirmaron que la mayoría de los adolescentes reportan una disfunción familiar moderada y un rendimiento académico medio.
Igualmente se encontró que la mayoría de ellos no presentó conductas externalizadas aunque hay un porcentaje de ellos que sí los presento, especialmente según lo reportado por los profesores.

Los resultados encontrados en la presente investigación aportan evidencias sobre algunas variables que no estarían influidas por la funcionalidad familiar, como el rendimiento académico tal cómo se vio en los estudios de Lescano y Delgado, (2012), y Moreno, (2011); pero talvez la funcionalidad familiar si podría influir en otras variables emocionales de los escolares, lo que debería considerarse al plantear investigaciones futuras.

Las familias juegan un papel relevante en el desarrollo de las habilidades sociales de sus hijas e hijos a través de su interactuar diario, y el ambiente donde ellos o ellas van creciendo y desarrollándose como personas, y es así que los indicadores emocionales tales como la impulsividad, inseguridad, agresividad, timidez, y ansiedad son reflejados en actitudes y características sobreentendidas que los niños realizan al momento de ejecutar sus dibujos de la figura humana.

Cuando las relaciones familiares se caracterizan por una adecuada cohesión, adaptabilidad y comunicación, se tendrá un buen funcionamiento familiar ya que la socialización familiar fija una serie de conductas en los niños, al cual es probable que los que estan dentro de ello sean personas con una buena estabilidad emocional en el futuro. Por el contrario, cuando las familias son moderadamente funcionales $\mathrm{y} / \mathrm{o}$ disfuncionales y los niños crecen en un ambiente inadecuado por la presencia de conflicto, carencia de diálogo y deficiente apoyo a los miembros de la familia, pueden surgir problemas en los niños como, por ejemplo, problemas emocionales llevándoles a conductas inadaptables o incorrectas y dificultad en su aprendizaje, por lo tanto los padres de familia deben estar atentos para prevenir estos posibles riesgos de comportamniento en sus niños.

La funcionalidad familiar juega un rol importantisimo en el desarrollo emocional del niño que le favorezca el proceso de adaptación en la sociedad y que le genere mecanismos de defensa que le permita afrontar positivamente los conflictos de la vida en el futuro.

\section{CONCLUSIONES}

La funcionalidad familiar no se relacionan significativamente con los indicadores emocionales en los escolares, Institución Educativa Integral Blas Valera Pérez Chachapoyas, demostrado por los valores de la prueba estadística no paramétrica ji cuadrada: $\mathrm{X}^{2}=5.135 ; \mathrm{gl}=2 ; \mathrm{p}=0.882>0.05 ; \mathrm{Un}$ 
poco menos de la mitad de las familias de los escolares, presentan funcionalidad familiar moderada, seguidas de las familias disfuncionales; y solo una cuarta parte son funcionales; Los escolares, presentan predominantemente indicadores emocionales en un nivel bajo.

\section{REFERENCIAS BIBLIOGRÁFICAS}

Bolaño, D., Cortina, J., y Vargas, S. (2013). Funcionalidad familiar en niños con dificultades en el aprendizaje escolar en dos instituciones educativas públicas del municipio de Sincelejo. REVISALUD Unisucre ISSN: 2339 - 4072.

Castellón, S., y Ledesma, E. (2012). El funcionamiento familiar y su relación con la socialización infantil, proyecciones para su estudio en una comunidad suburbana de sancti spíritus. Cuba. En Contribuciones a las Ciencias Sociales, Julio 2012, www.eumed.net/rev/cccss/21/

Castillo, S. (2012) Indicadores emocionales de los niños escolares de 6 a 12 años, institución educativa primaria $N^{\circ} 18003$ Santa Rosa Chachapoyas. TESIS para obtener el título profesional de Licenciada en Enfermería. Chachapoyas. Amazonas. Perú.

Clavijo, et al. (2016). Funcionalidad familiar asociado al bajo rendimiento académico en escolares de 6 a 10 años de instituciones oficiales de Cartagena. Universidad de Cartagena. Facultad de Enfermería.

Garaigordobil, M. y Maganto, C. (2012) Valora problemas emocionales y de conducta en la infancia: un instrumento de identificación y prevención temprana. Facultad de Psicología. Universidad del País Vasco.

Graza, S. (2013). Relación entre funcionalidad familiar y nivel de violencia escolar en los adolescentes de la Institución Educativa Francisco Bolognesi Cervantes $n^{\circ} 2053$ Independencia. TESIS Para optar el Título Profesional de Licenciada de Enfermería.

Henao, M. (2006). Test de la figura humana: Indicadores Emocionales. Estudio del psicoanálisis y psicología.

Lescano, E., Delgado, A. (2012). Funcionamiento familiar y rendimiento académico en estudiantes de secundaria de la institución educativa 0292-Tabalosos. Marzo 2011 y abril 2012. TESIS: para optar el título profesional de licenciada en enfemería. Tarapoto, Perú. Universidad Nacional de San Martin.

Mio, W. (2016). Indicadores emocionales en niños de tres instituciones educativas de nivel inicial Chachapoyas 2015. TESIS para optar el título de enfermería. Universidad Nacional Toribio Rodríguez de Mendoza, Chachapoyas. Perú.

Moreno, J., y Chauta, L. (2011). Funcionalidad familiar, conductas externalizadas y rendimiento académico en un grupo de adolescentes de la ciudad de Bogotá. Universidad de San Buenaventura, sede Bogotá, - Colombia. Psychologia. Avances de la disciplina. Recuperado de: $<$ http://www.redalyc.org/articulo.oa?id=2 97225770006> ISSN 1900-2386

Organización Mundial de la Salud, (2017). Concepto de la familia según la OMS. Recuperado de:http:/cheqoo.blogspot.pe/2009/10/con cepto-de-familia-segun-la-oms.htm

Ramírez, J. (2012). Indicadores emocionales del test del dibujo de la figura humana de Koopitz en niños y niñas con TDAH de la ciudad de Manizales. Candidata a Magíster en Desarrollo Infantil. Universidad de Manizales, Colombia.

Rangel, J. (2014). Funcionalidad familiar en la adolescente embarazada. Revista de la facultad de medicina: Medigraphic, com.

Valle, C. (2014). Teoría de la personalidad: modelo $d e \quad G r a y$. O b t e $\mathrm{n} \mathrm{i} \mathrm{d} \mathrm{o} \mathrm{d} \mathrm{e}$ http:/terapiainfantilyjuvenil.blogspot.pe/ 2014/12/teoria-de-la-personalidad-odelode-gray.html

Zaldívar, D., (s.f). Funcionamiento familiar s a lud able $R$ e cu perado e n : http://www.sld.cu/galerias/pdf/sitios/reha bilitaciontemprana/funcionamiento_famil iar 